\title{
SIMILITUDE ULTRASTRUCTURALE DES SPERMATOZOIDES DE QUELQUES CYCLOPHYLLIDEA
}

\author{
BA C.T.*, MARCHAND B.*
}

Summary : UltRASTRUCTURAL SIMILARITY OF THE SPERMATOZOA OF SOME CYCLOPHYLLIDEA

The mature spermatozoa of Cotugnia polyacantha, Raillietina (R) tunetensis, Inermicapsifer madagascariensis and Avitellina centripunctata have an almost identical ultrastructural organization. They are tapered at both extremities. Their anterior extremity exhibits an apical cone of electron dense material and one or two helicoidal crested-like bodies. Their cortical microtubules are spiralized and are seen, in longitudinal and cross sections, as continuous, dense, submembranous material. Their nucleus is a cord of dense chromatin, coiled in a spiral around the $9+1 "$ " pattern axoneme. The cytoplasm is moderately dense to electron lucent and exhibits irregularly spaced walls of proteinaceous material and a fine periaxonemal sheath of dense material. In this work we show that the Anoplocephalidae are close to the Davaineidae by the characters of the spermatozoon and that Inermicapsifer should be put back among the Davaineidae, according to the opinion of Joyeux \& Baer (1961) and come into conflict with other works, more particularly Schmidt (1986)

KEY WORDS : Cotugnia polyacantha. Raillietina (R) tunetensis. Inermicapsifer madagascariensis. Avitellina centripunctata. Anoplocephalidae. Davaineidae. Spermatozoon. Ultrastructure.

\section{INTRODUCTION}

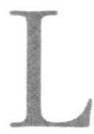

e spermatozoïde d'un cestode est généralement filiforme, dépourvu de mitochondrie et présente un cytoplasme transparent à moyennement opaque aux électrons, un ou plusieurs corps en crête à son extrémité antérieure (Bâ et al., 1991), des microtubules corticaux pouvant être ou non spiralés et un ou deux axonèmes de type $9+" 1 "$. Les études spermatologiques peuvent aider à la compréhension de la phylogenèse des Cestodes car l'absence de mitochondrie dans leurs spermatozoïdes les isole de l'ensemble des Plathelminthes. Par ailleurs, les microtubules corticaux sont torsadés uniquement chez les Cyclophyllidea, mais parallèles entre eux chez tous les autres Cestodes (Ehlers, $1985 a$ et $b$; Brooks, 1989 ; Justine, 1991). Dans diverses études nous nous sommes intéressés au contenu du cytoplasme spermatique. Nous avons ainsi pu mettre en évidence chez différentes espèces de Cestode (Tableau I) une gaine péri-axonémale de matériel opaque aux électrons (Bâ et Marchand, $1992 a ; 1993 b, c$, e et g), du

\footnotetext{
* Laboratoire de Parasitologie, Département de Biologie animale, Faculté des Sciences et Techniques, université Ch. A. Diop de Dakar, Dakar, Sénégal.
}

\section{Résumé}

Les spermatozoïdes mûrs de Cotugnia polyacantha, Raillietina $(R \mid$ tunetensis, Inermicapsifer madagascariensis et Avitellina centripunctata ont une organisation ultrastructurale quasi identique. Is sont effi lés à leurs deux extrémités. Leur extrémité antérieure présente un cône apical de matériel opaque aux électrons et un ou deux corps hélicoïdaux en crête. Leurs microtubules corticaux sont spiralés et apparaissent en coupes longitudinales et transversales sous la forme d'un matériel dense continu sous-membranaire. Leur noyau est un cordon de chromatine dense, enroulé en spirale autour de leur axonème de type $9+$ " " ". Leur cytoplasme est moyennement opaque à transparent aux électrons et présente des cloisons irrégulièrement espacées de matériel protéique et une fine gaine péri-axonémale de matériel dense. Dans ce travail nous montrons que les Anoplocephalidae et les Davaineidae sont proches par des caractères du spermatozoïde et que Inermicapsifer devrait être placé dans les Davaineidae, conformément à l'opinion de Joyeux et Baer (1961) et en opposition avec d'autres travaux, notamment Schmidt (1986).

MOTS CLES : Cotugnia polyacantha. Raillietina (R) tunetensis. Inermicapsifer madagascariensis. Avitellina centripunctata. Anoplocephalidae. Davaineidae. Spermatozoïde. Ultrastructure.

matériel dense amorphe (Bâ et Marchand, 1993f), des granules de matériel dense (Mackinnon et Burt, 1984 ; Bâ et al., 1991 ; Bâ et Marchand, $1992 b$ et c ; $1993 a$ et $d$ ), des plages de matériel transparent aux électrons (Bâ et Marchand, 1993d) et des cloisons irrégulièrement espacées de matériel protéique (Bâ et Marchand, 1993c, e et $g$ ). Dans la présente note, nous comparons l'ultrastructure des spermatozoïdes de deux Davaineidae, Cotugnia polyacantha et Raillietina $(R)$ tunetensis et de deux Anoplocephalidae, Avitellina centripunctata et Inermicapsifer madagascariensis.

\section{MATERIEL ET METHODES}

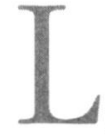

es specimens de Cotugnia polyacantha Führmann 1909 et Raillietina (R) tunetensis Joyeux et Houdemer 1928 ont été récoltés respectivement dans l'intestin des tourterelles, Streptopelia vinacea (Vinaceous Dove) et $S$. senegalensis (Laughing Dove). Par contre, Inermicapsifer madagascariensis Davaine 1870 et Avitellina centripunctata (Rivolta 1874) Grough 1911 ont été prélevés respectivement dans le tube digestif de Arvicanthis niloticus (rongeur) et des petits ruminants (moutons 
BA C.T., MARCHAND B.

\begin{tabular}{|c|c|c|c|c|c|c|c|}
\hline $\begin{array}{c}\text { Famille } \\
\text { Genre espèce } \\
\text { (référence) }\end{array}$ & $\begin{array}{l}\text { Corps } \\
\text { en crête }\end{array}$ & $\begin{array}{l}\text { Granule } \\
\text { dense }\end{array}$ & $\begin{array}{l}\text { Plages de } \\
\text { matériel clair }\end{array}$ & $\begin{array}{l}\text { Cloisons } \\
\text { de matériel } \\
\text { protéique }\end{array}$ & $\begin{array}{l}\text { Gaine péri- } \\
\text { axonémale }\end{array}$ & $\begin{array}{l}\text { Organe } \\
\text { parutérin }\end{array}$ & $\begin{array}{l}\text { Capsule } \\
\text { ovifêre }\end{array}$ \\
\hline \multicolumn{8}{|l|}{$\begin{array}{l}\text { Anophocephalidae } \\
\text { Triuterina sp (Baer, 1927) }\end{array}$} \\
\hline $\begin{array}{l}\text { Monøecostus americamus } \\
\text { (Mackinnon \& Burt, 1984) }\end{array}$ & & + & & & & & \\
\hline $\begin{array}{l}\text { Thysaniezia ovilla } \\
\text { (Bâ et al., 1991) }\end{array}$ & $\begin{array}{l}+ \\
2\end{array}$ & + & & & & + & \\
\hline $\begin{array}{l}\text { Stilesia globipunctata } \\
\text { (Bâ \& Marchand, 1992a) }\end{array}$ & + & & & & + & + & \\
\hline $\begin{array}{l}\text { Moniezia expansa } \\
\text { (Bâ \& Marchand, 1992c) }\end{array}$ & $\begin{array}{l}+ \\
2\end{array}$ & + & & & & & \\
\hline $\begin{array}{l}\text { M. benedeni } \\
\text { (Bâ \& Marchand, 1992c) }\end{array}$ & $\begin{array}{l}+ \\
2\end{array}$ & + & & & & & \\
\hline $\begin{array}{l}\text { Mathevotaenia herpestis } \\
\text { (Bâ \& Marchand, 1993b) }\end{array}$ & + & & & & + & & \\
\hline $\begin{array}{l}\text { Avitellina centripunctata } \\
\text { (Bâ \& Marchand, 1993b) }\end{array}$ & + & & & + & + & & \\
\hline $\begin{array}{l}\text { Aporina delafondi } \\
\text { (Bâ \& Marchand, 1993d) }\end{array}$ & $\begin{array}{l}+ \\
5\end{array}$ & + & + & & & & \\
\hline $\begin{array}{l}\text { Inermicapsifer guineensis } \\
\text { (Bâ \& Marchand, 1993g) }\end{array}$ & $\begin{array}{l}+ \\
2\end{array}$ & & & + & + & & + \\
\hline $\begin{array}{l}\text { I. madagascariensis } \\
\text { (Bâ \& Marchand, 1993g) }\end{array}$ & $\begin{array}{l}+ \\
2\end{array}$ & & & + & + & & + \\
\hline $\begin{array}{l}\text { Davaineidae } \\
\text { Davainea sp (Baer, 1927) }\end{array}$ & & & & & & & + \\
\hline $\begin{array}{l}\text { Raillietina }(R) \text { tunetensis } \\
\text { (Bâ \& Marchand, 1993e) }\end{array}$ & $\begin{array}{l}+ \\
2\end{array}$ & & & + & + & & + \\
\hline $\begin{array}{l}\text { Cotugnia polyacantha } \\
\text { (présent travail) }\end{array}$ & $\begin{array}{l}+ \\
2\end{array}$ & & & + & + & & + \\
\hline $\begin{array}{l}\text { Hymenolepididae } \\
\text { Hymenopelis nana } \\
\text { (Bâ \& Marchand, 1992b) }\end{array}$ & $\begin{array}{c}+ \\
12\end{array}$ & + & & & & & \\
\hline $\begin{array}{l}\text { Retinometra serrata } \\
\text { (Bâ \& Marchand, 1992b) }\end{array}$ & $\begin{array}{l}+ \\
6\end{array}$ & + & & & & & \\
\hline
\end{tabular}

Tableau I. - Quelques caractères spermatiques et morphologiques des Cyclophyllidea cités dans le présent travail. Le signe plus et les chiffres indiquent respectivement la présence du caractère indiqué et le nombre de corps en crête.

et chèvres). Ces cestodes ont ensuite été gardés vivants et actifs dans du sérum physiologique (solution de $\mathrm{NaCl}$ à $9 \%$ ). Les appareils génitaux mâles et femelles ont été prélevés sous la loupe, maintenus dans le glutaraldéhyde pendant environ 24 heures, rincés une nuit dans du tampon cacodylate de sodium, post-fixés au tetroxide d'osmium froid à $1 \%$ pendant une heure, déshydratés par l'éthanol et l'oxide de propylène, puis inclus dans l'épon. Les coupes ultrafines ont été réalisées à l'ultramicrotome Porter-Blum MT1, puis contrastées par l'acétate d'uranyle et le citrate de Plomb. Les observations ont été faites aux microscopes électroniques, Siemens Elmiskop 101 et JEOL 100 C X II.

\section{OBSERVATIONS}

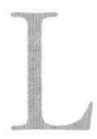

es spermatozoïdes mûrs de Cotugnia polyacantha, Raillietina (R.) tunetensis, I. madagascariensis et $A$. centripunctata sont effilés à leurs deux extrémités (Figs. 1 et 6 ). Ils mesurent entre 0,1 et $0,6 \mu \mathrm{m}$ de large et présentent à leur extrémité antérieure un cône apical de matériel opaque aux électrons (Fig. 1) et un (Fig. 9) ou deux corps hélicoïdaux en crête décalés longitudinalement l'un par rapport à l'autre (Figs. 1, 7 et 8). Ainsi, en coupes transversales et selon le niveau de la section, il apparaît une ou 

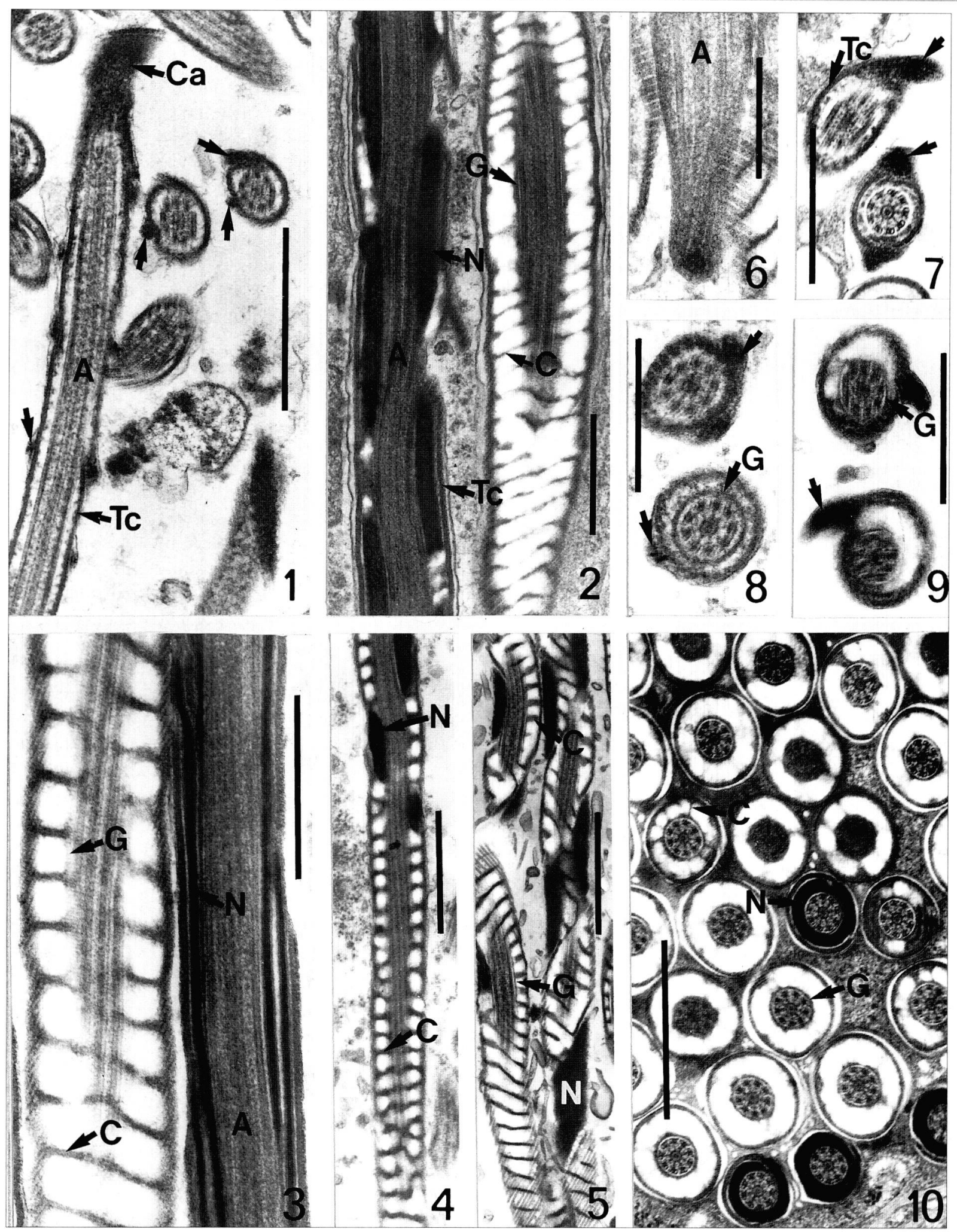

crête. Barre $=1 \mu \mathrm{m}$. Fig. 2. - Coupes longitudinales du spermatozoïde mûr de C. polvacantha. Barre = $1 \mu \mathrm{m}$. Fig. 3. - Coupes longitudinales du spermatozoïde mûr de $R$. (R.) tunetensis. Barre $=1 \mu \mathrm{m}$. Fig. 4. - Coupe longitudinale du spermatozoïde mûr de $I$. madagascariensis. Barre $=1 \mu \mathrm{m}$. Fig. 5 . - Coupes longitudinales du spermatozoïde mûr de Avitellina centripunctata. Barre $=1 \mu \mathrm{m}$. Fig. 6. - Coupe longitudinale de l'extrémité postérieure du spermatozoïde mûr de C. polyacantha. Barre $=1 \mu \mathrm{m}$. Fig. 7. - Coupe transversale de l'extrémité antérieure du spermatozoïde mûr de $R$. ( $R$.) tunetensis. Les flèches indiquent les corps en crête. Barre $=0,5 \mu \mathrm{m}$. Fig. 8. - Coupes transversales de l'extrémité antérieure du spermatozoïde mûr de 1 . madagascariensis. Les flèches indiquent les corps en crête. Barre $=0,5 \mu \mathrm{m}$. Fig. 9. - Coupe transversale de l'extrémité antérieure du spermatozoïde mûr de Avitellina centripunctata. La flèche indique la présence d'un seul corps
zoïde mûr de Cotugnia polyacantha. Barre $=1 \mu \mathrm{m}$.

A, axonème : C, cloison intracytoplasmique de matériel dense : Ca, cône apical : G, gaine péri-axonémale de matériel dense : V, noyau : Tc, microtubules 
deux crêtes latérales (Figs. 1, 7 et 8). Leurs microtubules corticaux sont spiralés et apparaissent en coupes longitudinales (Figs. 1 à 6) et transversales (Figs. 1, 7, 8 et 9) sous la forme d'un matériel dense continu sousmembranaire. Leur axonème, du type 9 + "1" (Figs. 7 et 10), est entouré d'une fine gaine de matériel dense (Figs. 2, 3, 5, 8, 9 et 10). Leur cytoplasme est moyennement opaque (Fig. 6) à transparent aux électrons et contient des cloisons irrégulièrement espacées de matériel opaque aux électrons (Figs. 2, 3, 4, 5 et 10). Ces derniers sont disposés perpendiculairement à l'axonème et relient la gaine de matériel peri-axonémale aux microtubules corticaux (Figs. 2 à 5). Leur noyau est un simple cordon de matériel dense enroulé en une spirale serrée autour de l'axonème (Figs. 2 à 5). Il apparait ainsi en coupes transversales, selon le niveau de la section, sous la forme d'un croissant ou d'un anneau (Fig. 10).

\section{DISCUSSION}

L

'ordre des Cyclophyllidea comprend 6 familles dont les Davaineidae caractérisés par la présence d'un scolex muni d'un rostre et de crochets en forme de marteau et les Anoplocephalidae (Schmidt, 1986). Ces derniers sont inermes et ont un cycle évolutif comportant un acarien coprophage de l'ordre des Sarcoptiformes, du sous-ordre des Oribatides chez lequel se forme une larve cysticercoïde infestante pour l'hôte définitif du parasite (Euzeby, 1966). Un des problèmes posés par l'anatomie comparée des Cestodes a été de savoir si les Davaineidae dérivaient ou non des Anoplocephalidae. Baer (1927), se basant sur les ressemblances anatomiques et biologiques, la position systématique des hôtes et la théorie des mutations, a tenté d'établir des relations phylogénétiques entre différents genres de Cestodes en particulier entre les Triuterina (Anoplocephalidae) et les Davainea (Davaineidae). Chez ces deux genres, les conduits sexuels passent entre les canaux excréteurs et l'utérus tubulaire présente une cavité accessoire. Selon Baer (1927) les Davaineidae proviendraient des Anoplocephalidae et les Triuterina seraient le chaînon qui rattacherait ces deux familles. Certes, la présence de cloisons intracytoplasmiques de matériel opaque aux électrons et d'une gaine péri-axonémale de matériel dense dans les spermatozoïdes de Avitellina centripunctata, Inermicaspifer madagascariensis, Cotugnia polyacantha et Raillietina $(R)$ tunetensis (tableau I) montre des affinités phylogénétiques étroites entre les Anoplocephalidae et les Davaineidae, mais nous pensons que l'étude anatomique comparée d'un nombre plus important de genres est nécessaire pour savoir laquelle de ces deux familles est la plus ancienne.
Un autre problème important est de savoir si les Inermicapsifer sont des Anoplocephalidae ou des Davaineidae ? La difficulté vient du fait qu'ils présentent des caractères de chacune de ces deux familles. Spasskii (1951), Yamaguti (1959), Euzeby (1966) et Schmidt (1986) ont pris comme argument leur caractère inerme et les ont placés parmi les Anoplocephalidae. Joyeux et Baer (1961) par contre, ont considéré la présence dans leurs anneaux gravides de capsules ovifères contenant de nombreux œufs comme chez les Davaineidae et les ont classés dans les Davaineidae. Dans le présent travail, nous montrons que le spermatozoïde de Cotugnia polyacantha présente une gaine péri-axonémale de matériel dense, des cloisons intracytoplasmiques de matériel opaque aux électrons et deux corps hélicoïdaux en crête à son extrémité antérieure. Il en est de même des spermatozoïdes de Raillietina (R.) tunetensis (Bâ et Marchand, 1933e), Inermicapsifer guineensis et I. madagascariensis (Bâ et Marchand, 1933g). A notre connaissance, un tel spermatozoïde n'a jamais été décrit chez les autres Anoplocephalidae (tableau I). Ainsi, bien qu'étant inerme nous pensons que les Inermicapsifer sont des Davaineidae.

La formation de capsules ovifères, d'organes parutérins etc. dans différents genres de Cestodes a été interprétée par Führmann (1907) et Baer (1927) comme de simples phénomènes de convergence. Brooks et al., (1991) cependant considèrent les organes parutérins comme une synapomorphie caractérisant un groupe monophylétique de Cyclophyllidea. Par ailleurs, nos observations semblent montrer que les Cestodes qui ont des capsules ovifères d'origine utérine contenant un (Cotugnia polyacantha) ou plusieurs oufs (Inermicapsifer madagascariensis, I. guineensis et Raillietina (R.) tunetensus) présentent le même type de spermatozoïde.

\section{REFERENCES}

BA C.T., Marchand B. : Ultrastructural particularities of the spermatozoon of Stilesia globipunctata (Cestoda) parasite of the small intestine of sheep and goats in Senegal. Journal of Submicroscopic Cytology and Pathology, 1992a, 24, 29-34.

Ba C.T., Marchand B. : Reinvestigation of the ultrastructure of spermiogenesis and the spermatozoon of Hymenolepis nana (Cestoda, Cyclophyllidae) parasite of the small intestine of Rattus rattus. Molecular Reproduction and Development, 1992 b, 33, 39-45.

BA C.T., MARChAND B. : Ultrastructural study of the spermatozoa of Moniezia expansa Rudolphi, 1810 and M. benedeni Moniez, 1879 (Cestoda, Cyclophyllidea, Anoplocephalidae). Annales de Parasitologie bumaine et comparée, 1992c, 67, 111-115. 
BA C.T., MARChand B. : Ultrastructure of the Retinometra serrata spermatozoon (Cestoda) intestinal parasite of turtle-doves in Senegal. Journal of Submicroscopic Cytolology and Pathology, 1993a, 25, 233-238.

BA C.T., Marchand B. : Ultrastructure of spermiogenesis and the spermatozoon of Mathevotaenia herpestis (Cestoda) intestinal parasite of Atelerix albiventris in Senegal. Acta Zoologica, 1993b, 75, sous presse.

BA C.T., MARCHAND B. : Ultrastructure of the spermatozoon of Avitellina centripunctata (Cestoda, Cyclophyllidea), a parasite of the small intestine of cattle in Senegal. Acta Zoologica, 1993c, 75, sous presse.

Ba C.T., Marchand B. : Ultrastructure of spermiogenesis and the spermatozoon of Aporina delafondi (Cyclophyllidea, Anoplocephalidae) parasite of the intestine of turtle doves in Senegal. International Journal for Parasitology, $1993 d$ (sous presse).

Ba C.T., Marchand B. : Ultrastructure of spermiogenesis and the spermatozoon of Raillietina (Railliteina) tunetensis (Cyclophyllidea, Davaineidae) intestinal Parasite of Turtle doves in Senegal. International Journal for Parasitology, 1993e (sous presse).

BA C.T., Marchand B. : Ultrastructure of the spermatozoon of Sandonella sandoni (Cestoda, Proteocephalidea, Sandonellinae) intestinal Parasite of Heterotis niloticus (Fish, Teleost). International Journal of Invertebrate Reproduction and Development, $1993 f$ (sous presse).

BA C.T., Marchand B. : Comparative ultrastructure of the spermatozoa of Inermicapsifer guineensis and I. madagascariensis (Cestoda, Anoplocephalidae, Inermicapsiferinae) intestinal parasites of rodents in Senegal. Canadian Journal of Zoology, $1993 \mathrm{~g}$ (soumis pour publication).

Ba C.T., Marchand B., Mattei X. : Demonstration of the orientation of the Cestodes spermatozoon illustrated by the ultrastructural study of spermiogenesis and the spermatozoon of a Cyclophyllidea : Thysaniezia ovilla Rivolta, 1874. Journal of Submicroscopic Cytology and Patbology, 1991, 23, 605-612.

BAER J.G. : Monographie des Cestodes de la famille des Anoplocephalidae. Bulletin biologique de France et de Belgique, 1927, Suppl. 10, 241 p..

Brooks D.R., Hoberg E.P., Weekes P.J. : Preliminary phylogenetic systematic analysis of the major lineages of the Eucestoda (Platyhelminthes : Cercomeria). Proceedings of the Biological Society of Washington, 1991, 104, 651-668.

EHLERs U. : Das phylogenetischen system der plathelminthes. Gustav Fischer Verlag, Stuttgart, 1985a.

Ehlers U. : Phylogenetic relationships within the Platyhelminthes. In The Origins and Relationships of Lower Invertebrates. Edited by Conway Morris S., Georges J.D., Gibson R. and Platt H.M., Oxford University Press, Oxford, 1985b, p. 143-158.

EuzeBy J. : Maladies dues aux Plathelminthes. T. 2. Fasc. 1 : Cestodoses. Cestodes, Vigot frères, Ed., Paris, 1966, 236.

Führmann O. : Die Systematik der Ordnung der Cyclophyllidea. Zool. Anz., 1907, 32, 289-297.

Joyeux C., Baer J.G. : Classe des Cestodes. In Traité de
Zoologie, T. 4, P.P. Grassé Ed., Masson et Cie, Paris, 1961, 347-650.

Justine J.-L. : Phylogeny of parasitic Platyhelminthes : a critical study of synapomorphies proposed on the basis of the ultrastructure of spermiogenesis and spermatozoa. Canadian Journal of Zoology, 1991, 69, 1421-1440.

Mackinnon B.M., Burt M.D.B. : The comparative ultrastructure of spermatozoa from Bothrimonus stirionuis Duv. 1842 (Pseudophyllidea), Pseudanthobothrium hanseni Baer, 1956 (Tetraphyllidea) and Monoecocestus americanus Stiles, 1895 (Cyclophyllidea). Canadian Journal of Zoology, 1984, 62, 1059-1066.

SCHмiDT G.D. : CRC handbook of tapeworm identification. CRC Press, Boca Raton, Florida, 1986, 675 p.

SpAsskil A.A. : Anoplocephalata. Essentials of Cestodology. Vol. 1. Ed. Skrjabin K.I. Akad. Nauk., SSSR, Moskva. (English Translation by Israël Program for Scientific Translations), 1951, $783 \mathrm{p}$.

Yamaguti S. : Systema Helminthum. Vol. 2. The Cestodes of Vertebrates. Interscience Publishers, New York, 1959, $860 \mathrm{p}$. 